\title{
Alter
}

Revue de phénoménologie

21 | 2013

La Vie

\section{Vers une biologie privative}

Réponse à Pierre Rodrigo

Renaud Barbaras

\section{(2) OpenEdition}

Journals

Édition électronique

URL : http://journals.openedition.org/alter/881

DOI : $10.4000 /$ alter.881

ISSN : 2558-7927

Éditeur :

Association ALTER, Archives Husserl (CNRS-UMR 8547)

Édition imprimée

Date de publication : 1 novembre 2013

Pagination : 267-275

ISBN : 978-2-95-223749-9

ISSN : 1249-8947

Référence électronique

Renaud Barbaras, « Vers une biologie privative », Alter [En ligne], 21 | 2013, mis en ligne le 01 juin 2019, consulté le 07 juillet 2019. URL : http://journals.openedition.org/alter/881 ; DOI : 10.4000/alter.881 


\section{VERS UNE BIOLOGIE PRIVATIVE. RÉPONSE À PIERRE RODRIGO}

Renaud Barbaras

Je dois concéder, à la lecture de la conclusion du texte de Pierre Rodrigo, que le titre de mon dernier ouvrage, La vie lacunaire, est mal choisi car la vie comme telle ne peut en effet être lacunaire : elle est au contraire surpuissante et donc dépourvue de la moindre faiblesse ontologique. Seul le vivant est lacunaire mais, en revanche, il l'est de manière constitutive car c'est précisément ce qui le définit comme vivant. Afin de justifier cette conclusion, je renvoie au début du chapitre IV de la troisième partie de mon ouvrage intitulé Dynamique de la manifestation ${ }^{1}$.

J'ai établi que le mouvement du sujet devait être caractérisé comme un vivre, autrement dit qu'il renvoyait à un sens originaire de la vie plus profond que le partage de la vie transitive et de la vie intransitive, de l'erleben et du leben. Cela signifie qu'il n'y a pas d'expérience qui ne s'enracine dans un être en vie mais pas non plus de vie, aussi fruste soit-elle, qui n'enveloppe une expérience et soit donc, en ce sens, intentionnelle. C'est là une autre manière de dire que l'appartenance du sujet au monde et sa puissance de le faire paraître ne font pas alternative mais sont au contraire l'envers l'une de l'autre car elles puisent à la même source. L'appartenance correspond au versant de l'être-en-vie, la phénoménalisation à celui du vivre transitif ; l'une et l'autre sont des expressions de cette vie originaire qui nomme l'être même du sujet. Cependant, l'accès à une telle vie n'est possible qu'à la condition de se défaire de la conception dominante de la vie, caracté-

\footnotetext{
${ }^{1}$ R. Barbaras, Dynamique de la manifestation, Paris, Vrin, 2013.

(C) Librairie Philosophique J. Vrin, Paris, 2013. http://www.vrin.fr. Extrait publié avec l'aimable autorisation des éditions Vrin.
} 
risée par deux présupposés solidaires. D'une part, la vie serait centrée sur le vivant, elle en serait une propriété ou une modalité, corrélative d'un certain degré d'organisation, loin que le vivant procède de la vie. D'autre part, et par là-même, la vie serait négation de cette négation potentielle qu'est la mort, comme retour aux lois de la nature inerte (non-vivante) : elle se réduirait à la réalisation des conditions qui permettent d'échapper à la mort, elle serait conservation de soi, survie. Or, nous avons montré que cette découverte d'un vivre originaire et neutre à la fois exigeait et permettait une neutralisation de ce que nous avons nommé l'ontologie de la mort, à savoir la démarche consistant à aborder la vie à partir de ce qui n'est pas elle et la menace, à la saisir du point de vue de ce qui la nie plutôt que de celui de son propre mode d'affirmation. Ainsi, le vivre originaire par lequel nous avons caractérisé l'étant que nous sommes n'est compréhensible que si l'on aborde la vie à partir d'elle-même plutôt que de ce qui la menace et, par voie de conséquence, le vivant à partir de la vie et non l'inverse. En effet, si en centrant la vie sur le vivant, on est nécessairement conduit à la comprendre comme négation de la mort, à l'inverse, dès l'instant où on la libère de cette référence à son autre, on cesse de la subordonner au vivant pour comprendre au contraire celui-ci comme une modalité de la vie. Telle est la conclusion à laquelle la phénoménologie de la corrélation nous a conduite : ce n'est pas parce que nous sommes des vivants (des organismes) que nous sommes en vie; c'est au contraire parce que nous sommes en vie, parce que nous avons reçu la vie en partage, que nous sommes des vivants. Il est impossible de comprendre comment un étant peut être en mouvement s'il n'est pas toujours déjà dans le mouvement, comment il peut être vivant, c'est-à-dire précisément accomplir un certain nombre de mouvements, s'il n'est pas déjà dans la vie, inscrit en elle. Autant dire que, comme règne ontologique singulier, le mouvement qui nous caractérise ne commence pas avec nous car, en vérité, il ne peut avoir commencé ; le mouvement est en nous dans la mesure où nous sommes en lui, nous le possédons dans la mesure où il nous possède. Ainsi, la vie que nous vivons n'est pas véritablement la nôtre au sens où nous n'en sommes ni la source, ni même, en vérité, le sujet : vivre c'est s'inscrire dans une vie qui nous précède et que nous reprenons pour ainsi dire à notre compte en lui donnant une inflexion singulière qui sera la marque de notre propre vie.

De cette vie qui nous précède, vie absolument originaire et anonyme, nous avons une idée assez précise et c'est même notre propre vie qui, selon un mouvement régressif qui a fait l'objet de notre seconde partie, nous y a initié. Nous avons montré en effet que 
l'appartenance constitutive du sujet signifie que notre mouvement s'inscrivait lui-même dans un archi-mouvement qui délivre le sens d'être véritable du monde: le sol ontologique d'appartenance d'un sujet dynamique ne peut être que de nature processuelle. Cet archimouvement n'est autre que celui par lequel le monde se fait monde, c'est-à-dire sort de l'indifférenciation en produisant des étants en son sein, en se multipliant au cœur de lui-même : le monde (se) mondifie en constituant une multiplicité d'étants quasi-individués dont l'unité est le monde mondifié comme totalité, sédiment de l'indivisibilité du fond au sein du multiple. Or, de même que notre mouvement n'est autre que celui d'un vivre, l'archi-mouvement dans lequel il s'insère et dont il procède relève d'une archi-vie. Telle est la vie dans laquelle nous sommes, dont nous héritons et que nous prolongeons : il s'agit précisément de cette archi-vie du monde, vie qui n'est encore la vie de personne mais qui sera la vie de tous et que décrit le procès de mondification. Dire que nous sommes en vie, ou encore que nous nous mouvons, c'est rigoureusement reconnaître que nous sommes insérés dans cette archi-vie du monde et que toute la puissance que nous avons de nous mouvoir provient de celle de cette archi-vie, autrement dit de la puissance mondifiante du fond. D'autre part, nous avons montré que le procès de mondification, l'archi-mouvement du monde, était en même temps un procès de manifestation, dès lors que l'individuation par délimitation dont il est l'artisan correspond à une première sortie de l'occultation, à un premier apparaître. Il est, de ce point de vue, légitime de parler d'une vie de la manifestation, non pas seulement en un sens encore métaphorique, afin de signifier que la manifestation est de nature dynamique, relève d'un procès, mais bien parce que ce procès est une vie et même le seul sens possible de la vie, bref qu'il n'y a de vie que comme vie de la manifestation. Notre vivre ne peut être l'opérateur de l'apparaître subjectif que dans la mesure où l'archi-vie à laquelle il renvoie est déjà de part en part un procès de phénoménalisation, à savoir la vie même de la manifestation.

S'il est vrai que notre vie renvoie tout entière à l'archi-vie du monde, toute la question est néanmoins de savoir comment elle en procède exactement, comment elle se singularise, se spécifie comme notre vie, étant entendu que cette ipséité (ou égoïté) naît de ce mode de spécification et s'épuise d'une certaine façon en lui. En effet, les étants intramondains, tout entiers pris dans le procès ontogénétique de l'archi-vie et déposés par lui, sont certes ce qu'ils sont mais ne sont pas vivants pour autant. Nous disposons désormais de la réponse à cette question. Elle réside dans la découverte d'une pluralité, préci- 
sément d'une dualité des modes d'individuation - comme si l'individuation même pouvait encore s'individuer - dualité qui renvoie à la différence radicale entre archi-mouvement et archi-événement, entre cosmologie et métaphysique. En effet, comme nous l'avons longuement établi, la singularité de notre mode d'être, qui est celui de ce que l'on nomme subjectivité, renvoie tout entière à l'absolue singularité, à l'unicité de l'archi-événement par lequel l'archi-mouvement se sépare de lui-même et se poursuit hors de lui-même, se voyant ainsi privé de sa surpuissance, en vue de se rejoindre ou de se retrouver lui-même. Le surgissement des vivants est immédiatement corrélatif de l'avènement de l'archi-événement, il procède tout entier de cette scission qui affecte l'archi-vie mais dont elle ne peut être la source ; il relève d'une individuation par séparation du procès du monde et non plus par circonscription au sein de ce que ce procès produit. C'est de cette scission que naît la singularité de notre vivre, c'est-à-dire précisément sa dimension proprement transitive. Bien entendu, il y a une forme de transitivité, et même d'hypertransitivité de la vie du monde, puisqu'elle consiste en un produire et ne vise donc rien puisqu'elle peut tout réaliser. C'est au contraire dans la mesure où notre vie est privée de cette puissance, où elle ne peut donc rien faire d'autre que tendre vers, qu'elle est susceptible de faire paraitre, bref capable de cette transitivité phénoménalisante qui caractérise son propre vivre. La vie intentionnelle, qui est la vie des vivants, est une vie ontogénétique dégradée, ou une archi-vie impuissantée : son pouvoir phénoménalisant naît d'une privation de la puissance onturgique du monde. Bref, s'il est vrai que, comme tout étant, nous provenons de l'archi-vie $\mathrm{du}$ monde, nous nous singularisons cependant, au même titre que tous les autres vivants, par le fait que nous en procédons en nous en séparant - ou plutôt en en étant séparés par l'archi-événement -, que notre rapport à cette archi-vie est marqué par la distance, distance dont la tentative de franchissement est exactement synonyme de phénoménalisation subjective ou secondaire.

Une telle conclusion ne peut manquer de surprendre. Elle signifie en effet qu'un étant n'est vivant que dans la mesure où il est séparé de l'archi-vie par l'archi-événement de la scission, que, par conséquent, c'est en raison d'un défaut de la vie en lui plutôt que de sa pleine présence qu'un étant est qualifié de vivant. Dire d'un étant qu'il est vivant, c'est affirmer non pas qu'il possède la vie mais qu'il ne la possède pas, en tout cas pas comme la possèdent les autres étants. Il faut néanmoins préciser le sens de ces formules. En vérité, les étants non-vivants ne possèdent pas la vie mais sont plutôt possédés par elle ; c'est parce qu'il sont de part en part dans la vie, c'est-à-dire pris 
dans le procès mondifiant dont ils sont les purs produits, qu'il ne sont justement pas en vie. À l'inverse, ne sont en vie, c'est-à-dire vivants et, en ce sens précis, possèdent la vie que les étants qui ne sont pas possédés par elle, de part en part pris en elle mais demeurent au contraire à distance de la vie et, en ce sens précis, ne la possèdent pas complètement ou tout entière. Au fond, les étants vivants ne peuvent avoir la vie qu'à la condition de ne pas l'être; ils ne sont susceptibles de reprendre à leur compte l'archi-vie du monde qu'à la stricte condition d'en être séparés, d'esquisser, à la faveur de la différence permise par l'archi-événement, l'ipséité et la subjectivité sans lesquelles il n'y a pas de possession pensable. Un étant n'est donc vivant qu'à la condition de ne pas se confondre avec l'archi-vie, de ne pas être pleinement la vie, d'avoir perdu la puissance de la vie du monde. C'est en raison de cette perte originelle, corrélative de l'archi-événement, qu'il va finalement perdre la vie, comme on dit, c'est-à-dire franchir l'abîme de l'archi-événement pour s'immerger dans la vie anonyme du monde. En d'autres termes, un vivant n'est vivant que s'il est mortel mais il n'est mortel que s'il est d'emblée privé de la vie en sa surpuissance et son éternité, bref séparé de l'archi-vie. C'est rigoureusement en ce sens qu'il est légitime d'affirmer qu'un vivant n'est vivant qu'en raison de l'absence plutôt que de la présence de la vie en lui. À bien y penser, ceci s'avère parfaitement cohérent si on veut bien se souvenir que la singularité de la vie des vivants repose sur leur transitivité phénoménalisante, bref sur leur intentionnalité. En ce sens d'ailleurs, s'il est absolument légitime de parler de vie à propos de l'archi-vie du monde, il ne l'est pas de parler de vivre: cette vie n'est pas un vivre car rien n'est vécu en elle, et rien n'est vécu en elle car tout est produit par elle. Lui manque pour ainsi dire l'écart entre la visée et la réalisation, c'est-à-dire finalement le désir, sans lesquels rien ne peut paraître, et toute notre démarche aura consisté au fond à prendre au sérieux cet écart, à le penser vraiment comme écart en le reconduisant à sa condition de possibilité dernière, qui n'est autre que l'archi-événement lui-même. Quoi qu'il en soit, si la singularité des vivants renvoie bien à leur vivre, à savoir à l'épreuve transitive du monde au sein de ce monde, il n'y a pas de vivre et donc pas de vivants sans la distance ontologique, la profondeur d'une faille que le vivre transitif vise à combler. Or il n'y a pas de distance ontologique sans une séparation qui met certains étants à l'écart du procès de mondification, autrement dit sans la perte en eux de la puissance de l'archi-vie du monde, sans une sorte de chute hors de la vie. En ce sens précis, c'est bien en raison d'une forme de privation de la vie, tout au moins de sa puissance constitutive qui est celle de 
l'archi-vie, que les vivants sont vivants. Le vivant est négation plutôt que pleine affirmation de la vie: il n'apporte la vie que s'il s'en déporte, ne la prolonge que dans la mesure où il s'en sépare ${ }^{2}$.

Cette conclusion appelle au moins deux remarques. Tout d'abord, nous pourrions résumer ce résultat en parlant de biologie privative. Encore faut-il préciser ce que nous entendons par là car une telle formule n'est pas sans évoquer la zoologie privative heideggérienne, qui a suscité de nombreux débats. Celle-ci signifie en substance qu'on ne peut accéder au mode d'être animal que sur le mode privatif à partir du mode d'être du Dasein. Selon la formule abondamment commentée, «La vie est un mode d'être spécifique, mais il n'est essentiellement accessible que dans le Dasein. L'ontologie de la vie s'accomplit sur la voie d'une interprétation privative; elle détermine ce qui doit être pour que puisse être quelque chose qui ne serait "plus que vie" $»^{3}$. Cette zoologie privative pourrait également être nommée biologie privative dès lors que, comme l'écrit Heidegger, c'est le mode d'être de la vie même qui est atteint privativement à partir du Dasein. On l'aura compris, notre perspective est aux antipodes exacts de celleci et possède donc un tout autre sens. Elle ne signifie pas en effet que les vivants ne peuvent être saisis que privativement à partir de l'homme mais, au contraire, que tous les vivants, y compris l'homme lui-même, renvoient à une privation au sein de l'archi-vie du monde. Ainsi, non seulement, bien sûr, notre approche est inverse de celle de Heidegger puisqu'elle pense l'homme, comme tous les autres vivants, à partir de la vie, précisément de l'archi-vie, au lieu de penser la vie des «simplement» vivants à partir de notre existence, mais encore elle confère à la privation un sens tout autre. Chez Heidegger, celle-ci n'a de statut que méthodologique ou heuristique au sens où elle indique une voie d'accès au vivant, sur le mode soustractif, qui ne veut préjuger en rien de la nature ou de l'essence de ce vivant et de ce qui le distinguerait vraiment du Dasein. Au contraire, nous conférons à la privation un sens métaphysique, qui donne en quelque sorte la clé de la nature des vivants, y compris de nous-mêmes. Loin d'exprimer une forme de prudence méthodologique ou d'aveu d'impuissance face à l'abyssale différence de l'animal, la voie privative est

\footnotetext{
${ }^{2}$ C'est là un autre point qui nous sépare radicalement de Michel Henry. En effet, alors que pour lui la vie absolue est tout entière présente dans la vie de l'affectivité, s'affirme en elle au point que celle-ci n'est que la vie absolue s'apportant elle-même et se rapportant à elle-même, pour nous, au contraire, la vie des vivants procède d'une négation de l'archi-vie, qui, en outre, est aussi transcendante que la vie absolue henryenne est immanente et aussi dynamique que celleci est finalement immobile.

${ }^{3}$ Heidegger, Sein und Zeit, trad. E. Martineau, p. 59.
} 
pour nous l'expression d'une privation effective, qui n'est autre que l'œuvre de l'archi-événement et qui est la condition même du mode d'exister singulier du vivant. L'accès aux vivants relève d'une voie privative dans la mesure exacte où ils sont affectés d'une privation en soi, celle de l'archi-vie, sur le mode de la séparation, et où leur être repose sur cette privation. Être vivant, de quelque vivant qu'il s'agisse, c'est être privé de la vie, précisément séparé de la vie par l'archi-événement et c'est un ce sens précis et radical que l'on peut reprendre à notre compte l'expression de Heidegger. Elle renvoie à la mise en évidence d'un archi-événement affectant l'archi-vie du monde en la séparant d'elle-même et donnant ainsi lieu à des vivants dont le mode d'être repose sur la privation de cette archi-vie; elle renvoie à l'articulation singulière d'une métaphysique et d'une cosmologie.

Dès lors, cette biologie privative doit également être distinguée de l'anthropologie privative par laquelle nous avions cru bon de distinguer, dans un ouvrage antérieur ${ }^{4}$, la singularité de notre mode d'exister. En effet, il s'agissait pour nous de montrer que l'intentionnalité devait être pensée, en sa transitivité phénoménalisante, sur la base d'une privation de la transitivité vitale, d'une négation de la profusion qui caractérise la vie des vivants et va jusqu'à la profondeur du monde, plonge en elle, alors que, quant à nous, nous ne pouvons en atteindre que l'être objet. Bref, le couple conscience-objet naîtrait d'une limitation au cœur d'une relation plus originaire, qui met en rapport la vie, telle qu'elle s'atteste au sein des vivants, et le monde lui-même. Cependant, même si elle représentait une première étape vers la biologie privative telle que nous venons de la caractériser, cette démarche avait pour défaut majeur de repousser le problème de la différence humaine, de le reporter pour ainsi dire d'un cran au lieu de le résoudre véritablement. En effet, penser l'homme privativement à partir des autres vivants c'est certes faire l'économie d'un attribut positif par lequel il se spécifierait et échapper ainsi à l'emprise d'une tradition métaphysique dominante. Mais en passant du positif au négatif, on n'accède pas à la raison de cette différence. Dire que l'homme se distingue des autres vivants parce qu'en lui la profusion vitale est comme suspendue ou refoulée, c'est encore lui conférer une place d'exception, même si elle ne repose plus sur un attribut mais sur une négation, et c'est s'interdire ainsi de prendre pleinement en charge sa continuité avec les autres vivants. Cela revient, par une sorte d'inversion, et finalement de position en miroir vis-à-vis de

${ }^{4}$ R. Barbaras, Introduction à une phénoménologie de la vie, Paris, Vrin, 2008. 
Heidegger, à faire de l'homme un vivant privé de vie, au lieu de faire du vivant un homme privé d'humanité. Par conséquent, comme nous le pressentions, la seule manière d'échapper à cette difficulté, à la lumière de certaines indications de Rilke (qui constituait sur ce point notre référence majeure), consistait à reporter la négation en reconnaissant une sorte de précession de cette privation humaine au sein de la vie même ou, tout au moins, chez d'autres vivants. On ne peut penser une privation de la vie en l'homme que sur fond d'une privation qui affecte la vie elle-même : le vie est d'une certaine façon niée en l'homme parce qu'elle se nie elle-même. Bref, la continuité ne pouvait être restaurée qu'à la faveur d'une généralisation de la privation.

C'est exactement ce pas que nous venons de franchir et, d'une certaine façon, ce livre n'a pas eu d'autre but que de répondre à la question de la possibilité de cette auto-négation, de se donner les moyens théoriques permettant de généraliser l'anthropologie privative sous la forme d'une zoologie privative. Il ne s'agit donc plus de penser l'homme comme privé de ce que possèdent d'autres vivants, à savoir une sorte de puissance intentionnelle supérieure, de pouvoir extatique les plongeant dans le monde, mais de penser tout vivant comme privé de quelque chose et de comprendre sa vie à partir de cette privation. On le voit, la position du problème appelait la réponse : cela dont tous les vivants sont privés ne doit plus relever du vivre qui les caractérise ; cela qui leur fait défaut ne peut être qu'une vie qui n'est pas la leur, à la fois plus que la leur par sa puissance ou sa profusion mais moins du point de vue de son pouvoir phénoménalisant - à savoir précisément l'archi-vie qui ne peut être que celle du monde et se confond avec sa dynamique mondifiante. La distinction du cosmologique et du métaphysique, condition de l'engendrement des vivants par privation de l'archi-vie et donc séparation de celle-ci en elle-même, est absolument commandée par le problème de la possibilité d'une anthropologie privative qui ne creuse pas à nouveau l'écart, même si c'est sur un mode négatif et non positif, entre l'homme et les autres vivants et échappe ainsi à l'humanisme. Tous les vivants, y compris l'homme, sont privés de vie, à savoir séparés de l'archi-vie et c'est exactement en quoi et pourquoi ils sont vivants. Cependant, on le voit, cette conclusion soulève immédiatement un nouveau problème, inverse du précédent, car en gagnant la continuité nous perdons ce que nous avions acquis, à savoir la différence. La question est en effet désormais de comprendre en quoi nous nous distinguons des autres vivants, en quoi, également privés de l'archi-vie, l'homme et l'animal peuvent néanmoins posséder des modes d'être très différents, ce qui est phénoménologiquement incontestable. 
Avant de répondre à cette dernière question, il nous faut, comme nous l'avions annoncé, faire une seconde remarque. Nous avons fait usage du terme biologie pour désigner ce qui concerne la vie des vivants mais, en vérité, ce ne peut être que par convention et, pour ainsi dire par défaut. Si on entend en effet par biologie une discipline qui s'intéresse aux vivants simplement vivants et à la vie de ces vivants, force est de conclure à l'impossibilité de la biologie. Comme nous l'avons justifié, la tentative de s'en tenir au vivant, c'est-à-dire d'en comprendre la vie, le mode d'être, à partir de sa structure propre que nomme le concept d'organisme, est vouée à l'échec. Le vivant n'est compréhensible qu'à partir d'une vie qui n'est pas la sienne, vie anonyme dont le véritable sujet est le monde et dont le vivre procède par privation, constituant l'organisme en et par son propre vivre. Or, si l'archi-vie n'est encore la vie de personne, le vivre des vivants est beaucoup plus qu'un simple être-en-vie pour autant qu'il se rapporte au monde en le phénoménalisant. La biologie est donc toujours déjà manquée, par défaut dans une cosmologie qui concerne une vie qui n'est encore la vie d'aucun vivant, et par excès dans une métaphysique, qui est en même temps une phénoménologie, pour laquelle tout vivant est déjà un sujet capable de phénoménalisation, pour laquelle tout vivant est au fond déjà un existant. Le pur vivant est introuvable : soit il n'est pas un vivant, soit il est déjà un sujet; soit il est encore immergé dans l'archi-vie, soit il fait paraître le monde. La biologie s'excède donc nécessairement de part et d'autre d'elle-même dans une cosmologie ou une phénoménologie, séparées par l'archiévénement que prend en charge la métaphysique. Ainsi, en toute rigueur, deux disciplines et deux seulement se confrontent à la question de la vie: la pensée de la vie proprement dite relève de la cosmologie, celle du vivant est du ressort d'une phénoménologie éclairée par la métaphysique. 